\title{
Inteligência estratégica em instituições de ensino superior
}

Alam de Oliveira Casartelli

Doutor em Comunicação Social. Professor da Faculdade de Administração, Contabilidade e Economia da PUCRS

Alziro César de M. Rodrigues

Doutor em Administração. Professor da Faculdade de Administração, Contabilidade e Economia da PUCRS

Hélio Radke Bittencourt

Doutorando em Geografia. Professor da Faculdade de Matemática da PUCRS

Vicente Garibotti

\section{Bacharel em Administração pela PUCRS}

Este artigo examina a adoção de práticas de Inteligência Estratégica (IE) em Instituições de Ensino Superior (IES), por meio da análise das informações mais utilizadas no apoio às decisões de gestão e da identificação de recursos e elementos relevantes para a melhoria da gestão da informação. O trabalho foi baseado em uma pesquisa desenvolvida junto a gestores de uma IES privada. Os resultados destacam a importância de trabalhar a cultura da IES na gestão de informações no sentido de uma maior eficácia no trabalho de IE, bem como a importância da gestão por indicadores para contribuir no aprimoramento desse processo.

Palavras-chave: Inteligência Estratégica; Gestão Estratégica; Tecnologia da Informação; Ensino Superior.

\section{Strategic intelligence in higher education}

This paper examines the adoption of practices of strategic intelligence in institutions of higher education through the 
analysis of key information that support management decisions and identification of relevant resources to improve information management. The work was based on a research developed with managers of a private higher education institution. The results of the exploratory study point out the importance of dealing with the culture of information management in institutions of higher education as to improve Strategic Intelligence work and also reinforces the importance of management through indicators for improvement of this process

Keywords: Strategic Intelligence; Strategic Management; Information Technology; Institutions of Higher Education.

Recebido em 19.10.2009 Aceito em 30.06.2010

\section{Introdução}

O acentuado crescimento no número de instituições de ensino superior (IES) privadas tem sido uma característica marcante no cenário do ensino superior brasileiro desde o final dos anos 90. De 1999 a 2007, o número de IES particulares experimentou um aumento de mais de $200 \%$, contra $30 \%$ de IES públicas e $16 \%$ de IES comunitárias, confessionais e filantrópicas (FIG. 1).

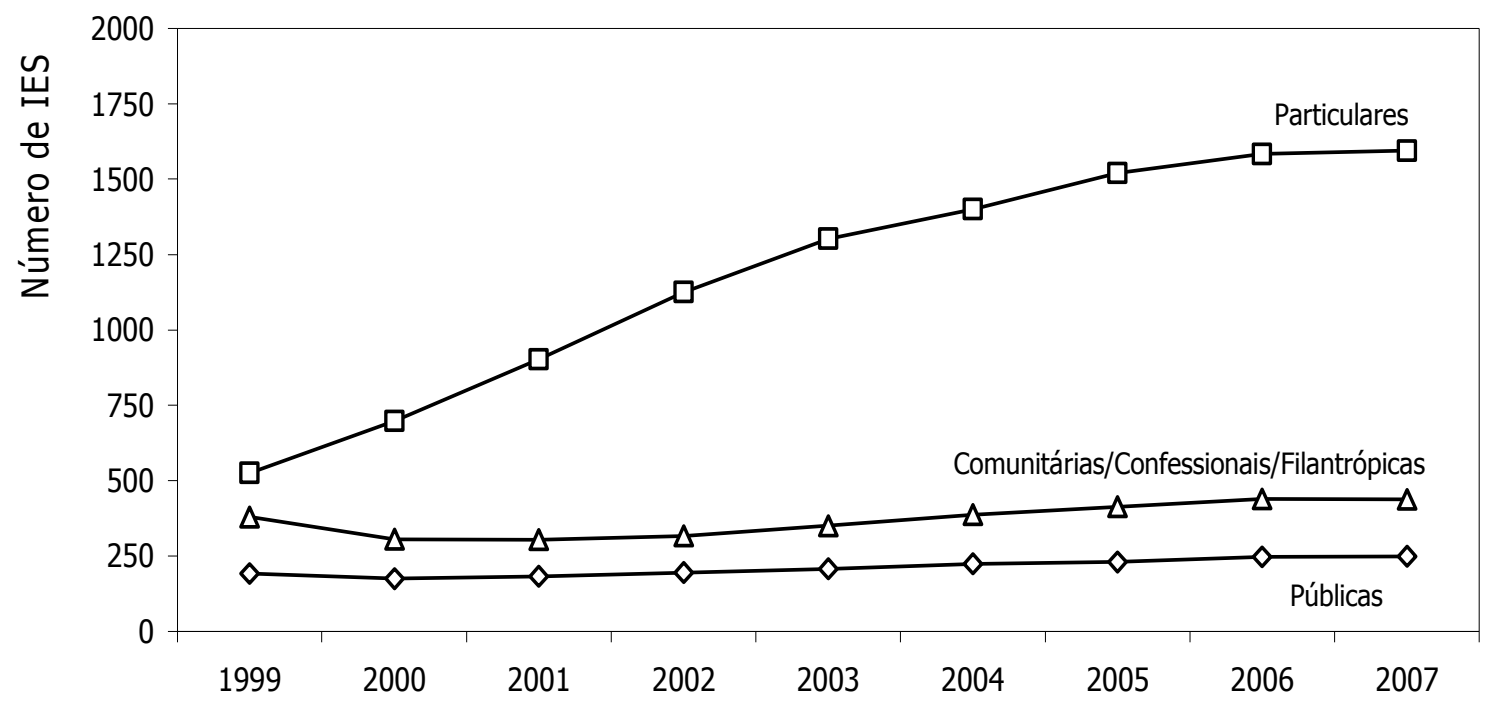

FIGURA 1 - Número de IES por natureza administrativa Fonte: INEP (1999-2007).

Essa constatação, aliada ao aumento nos níveis de ociosidade (conceito que traduz o número de vagas oferecidas e não preenchidas) em grande número de cursos de graduação, aponta para a necessidade de 
práticas mais efetivas de gestão no ensino superior privado, levando as IES a empreenderem ações e recursos para reforçar a captação de novos alunos e a buscarem diferenciar-se da concorrência. Ações baseadas em fortes campanhas de marketing e de comunicação, com investimentos em diferentes tipos de mídias, bem como decisões de redução nos valores das mensalidades, orientadas às classes sociais $D$ e $E$, têm sido cada vez mais presentes no setor do ensino superior (BRAGA; PEIXOTO; BOGUTCHI, 2001; MARTINS, 2008). Muitas são as reflexões sobre o posicionamento estratégico adotado pelas IES e sobre a qualificação do ensino superior como um todo, nesse contexto. Como consequência, as IES precisam tomar decisões que favoreçam a obtenção de melhores resultados e adotar práticas mais eficazes de gestão. Uma dessas práticas, ainda que de pouco uso no setor do ensino superior, é a Inteligência Estratégica (IE). A IE é essencial para as organizações já que, por meio do estudo de cenários e de análises de mercado, podem ser mais bem concebidas e implantadas ações que venham a assegurar os resultados pretendidos e a consecução dos objetivos traçados. Compreender as práticas de IE e sua importância para as organizações passa a ser condição fundamental para sua adoção.

Contribuindo para essa compreensão, o presente artigo orienta-se pelos seguintes objetivos: (a) analisar práticas de Inteligência Estratégica adotadas por IES; (b) identificar quais informações de apoio à decisão são mais utilizadas na gestão das IES; e (c) identificar recursos e elementos utilizados no processo de gestão da informação.

Para atingir tais objetivos, foi realizada uma pesquisa junto a 46 gestores de uma grande universidade privada gaúcha, desenvolvida em duas etapas. A primeira delas constituiu-se de uma pesquisa exploratória de caráter qualitativo, baseada em entrevistas de profundidade. A segunda etapa consistiu de uma pesquisa descritiva. Os resultados de cada fase são apresentados, analisados e discutidos neste artigo.

As seções subsequentes apresentam aspectos conceituais de Inteligência Estratégica, elementos da construção metodológica, a análise e a discussão dos resultados e, por fim, as conclusões do estudo.

\section{Inteligência Estratégica}

A Inteligência Estratégica (IE) está associada ao processo de análise de informações para construção de cenários macroambientais que impactam, de modo amplo, na gestão estratégica das organizações. Embora seja um conceito menos difundido que 0 de Inteligência Competitiva (IC), a IE mostra-se mais adequada a apoiar modelos de gestão no ensino superior, visto que a IC está mais direcionada às análises de informações de caráter mercadológico (FULD, 2007). O principal objetivo da IE é o de oferecer um suporte de informações para a adequada formulação e implementação de estratégias, utilizando, de forma mais eficaz, os recursos da organização e aprimorando o processo decisório (JANISSEK-MUNIZ; LESCA; FREITAS, 2006). 
A adoção de práticas de IE caracteriza a orientação da gestão de algumas organizações. A compreensão de tais práticas implica na abordagem de temas como: a) planejamento e gestão estratégica, já que as práticas de IE apoiam esses processos nas organizações; b) o conceito de Inteligência Competitiva (IC); c) a utilização de sistemas de informação; e d) o processo de tomada de decisões. A qualificação desses processos está no centro da prática de IE nas organizações e são discutidos a seguir.

O planejamento estratégico exerce papel essencial na gestão organizacional, centrado primariamente na identificação de fatores competitivos de mercado e de potencial interno, com vistas a atingir objetivos e implementar planos de ação que possam vir a gerar vantagem competitiva sustentável para a organização.

Para Oliveira (2001), o planejamento estratégico é um processo gerencial que permite aos gestores estabelecer objetivos a serem alcançados por meio de todas as estruturas da organização, tendo em vista sua integração ao seu ambiente e a obtenção de alto nível de qualidade em seu desempenho. Trata-se de um processo desenvolvido em etapas, que se inicia com o diagnóstico do posicionamento estratégico da organização em relação ao mercado, procurando (a) analisar o momento atual e identificar tendências internas e externas; (b) avaliar seu impacto para a organização; e (c) definir objetivos, metas e planos de ação. A fase seguinte é a da efetiva implementação, quando o que foi definido nos planos passa a ser executado.

O planejamento estratégico envolve a análise das atividades no macro e micro ambientes das organizações, a fim de apoiar o processo de tomada de decisões sobre os objetivos e estratégias. O desenvolvimento e a execução do plano são de responsabilidade dos executivos da organização, após sua aprovação e validação por parte da administração superior. Segundo Fischmann e Almeida (1990), o planejamento estratégico é um método que se inicia pela análise do ambiente de uma organização, criando a consciência de oportunidades e de ameaças, de seus pontos fortes e fracos para o cumprimento da sua missão e, por meio dessa consciência, estabelece o propósito de direção que organização deverá seguir.

As estratégias de mercado auxiliam as organizações a desenvolverem ações que beneficiem sua tomada de decisão e, por consequência, também são importantes aliadas da IE. Oliveira (2001) define a estratégia de mercado como uma ação estruturada e desenvolvida pela organização para alcançar, de forma adequada e preferencialmente diferenciada, os objetivos idealizados para o futuro, buscando o melhor posicionamento da empresa em seu ambiente. Seguindo lógica similar, Bateman e Snell (1998) sustentam que a estratégia de mercado identifica o conjunto de negócios, mercados ou setores em que a organização compete e a distribuição de recursos entre esses negócios. 
O planejamento estratégico e as estratégias de mercado, portanto, são baseados fortemente em análises de micro e macro ambientes e em diagnósticos internos, bem como na definição de cenários prospectivos de mercado. A prática de IE, por seu lado, auxilia nas análises e reflexões sobre as conjunturas e mudanças do ambiente que envolve as organizações. Tais análises e reflexões são a base de uma postura de gestão estratégica, orientada a antecipar-se às mudanças, ao invés de reagir a elas.

Diferindo do processo tradicional de planejamento estratégico, a gestão estratégica permite à organização adaptar sua maneira de ser, entendendo, aproveitando e antecipando as transformações em seu ambiente. Em oposição ao processo tradicional, a moderna gestão estratégica é uma forma de acrescentar novos elementos de reflexão e ação sistemática e continuada, conduzida e suportada pelos gestores da organização, a fim de avaliar a situação, elaborar um projeto e acompanhar e gerenciar os passos de implementação (WRIGHT; KROLL; PARNELL, 2000; HITT; IRELAND; HOSKISSON, 2002). As principais dificuldades de implementação das mudanças estratégicas não dizem respeito à qualidade do plano em $\mathrm{si}$, mas à forma pela qual se conduz o processo de pensar e renovar a organização e à maneira de implementar e acompanhar a execução.

Gestão estratégica é, portanto, um conceito muito mais amplo que o de planejamento estratégico. Engloba avaliações de diagnósticos, estruturação do processo de planejar e formular um propósito compartilhado para a organização, a escolha de estratégias, a fixação de metas e desafios, até a atribuição de responsabilidades para o detalhamento dos planos e projetos e para a condução e acompanhamento das etapas de sua implantação (COSTA, 2002). A gestão estratégica trata-se, de fato, de um processo sistemático, planejado, gerenciado, executado e acompanhado sob a liderança da alta administração da organização, envolvendo e comprometendo todos os gestores e colaboradores.

Por meio da contínua adequação da estratégia, da capacitação e da estrutura a gestão estratégica possibilita à organização enfrentar e antecipar-se às mudanças observadas ou previsíveis em seu ambiente externo, procurando assegurar seu crescimento, continuidade e sobrevivência.

Essencial à gestão estratégica é o processo de $\mathrm{IE}$, baseado na coleta, no tratamento e no uso da informação para apoiar as decisões que impactam no ambiente interno e no desempenho das organizações. Ao examinar esse aspecto, Alcará et al. (2009) apresentam uma discussão bastante completa sobre o compartilhamento da informação e do conhecimento no ambiente de pesquisa e desenvolvimento. Esses autores classificam esse processo como fonte de obtenção de vantagens competitivas.

Igualmente importante para compreensão da IE é o conceito de Inteligência Competitiva (IC). De acordo com a SCIP (2005), a IC é um 
processo sistemático e ético para reunir, analisar e administrar informações que podem afetar planos, decisões e operações de uma empresa. Prado (2007) concorda também com essa ideia, pois encara a IC como um processo sistêmico resultante da coleta, avaliação, análise e interpretação de dados e informações disponíveis no ambiente interno e externo, que possuam uma conexão com as características de uma determinada organização ou de áreas que subsidiam a tomada de decisão. Ou seja, a principal função da IC é apoiar a decisão gerencial.

Segundo Fuld (2007), a IC permite ao gestor visualizar as alterações do mercado, os avanços dos concorrentes e compreender a estratégia proposta, com a finalidade de definir ações antecipadas com esse conhecimento. Se o conceito de IC remete a um agir estratégico com foco no ambiente de negócios, mercado e concorrência, o conceito de IE, conforme visto anteriormente, surge na literatura para demarcar um campo de integração entre a IC e Gestão Estratégica.

Os processos de IE devem ser apoiados por sistemas de informação (SI) que facilitem a coleta, a análise e a disseminação dos dados e informações pela organização. De acordo com Freitas et al. (1997), sistemas de informação são mecanismos com a função de coletar, armazenar e distribuir informações para apoiar funções gerenciais e operacionais das organizações, que precisam estar totalmente inteiradas sobre seu ambiente. A busca de conhecimento sobre clientes, fornecedores, colaboradores, legislações e concorrentes é prática essencial para a sobrevivência em um mercado cada vez mais competitivo como o atual.

Na visão de Laudon (1999), um sistema de informações pode ser definido como um conjunto de componentes inter-relacionados trabalhando juntos para coletar, processar, armazenar, recuperar e distribuir informações com a finalidade de facilitar o planejamento, o controle, a coordenação, a análise e o processo decisório em empresas. A utilização de sistemas de informação é, portanto, fundamental na análise, organização e disseminação das informações que irão apoiar o processo de tomada de decisão nas organizações.

A tomada de decisão organizacional pode ser vista como o fechamento de todo esse ciclo. Após os esforços de coleta, tratamento e organização de informações gerenciais com o apoio de sistemas, por meio de um processo de $\mathrm{IE}$, os gestores dispõem de subsídios para escolher a melhor orientação estratégica para sua organização. De acordo com Vaitsman (2001), a tomada de decisão apoia-se nas informações disponíveis sobre um dado problema, de forma a propiciar ao decisor um razoável número de alternativas, dentre as quais deverá ser escolhida uma como a melhor ou a mais favorável. A tomada de decisão nada mais é, portanto, do que uma seleção entre alternativas de escolha com base em informações gerenciais (REZENDE; ABREU, 2003). Para a execução de uma tomada de decisão eficaz é preciso que sejam disponibilizadas informações adequadas e oportunas que possuam total relevância para o processo. 
Portanto, independentemente do nível organizacional, o uso inteligente da informação é fundamental para a tomada de decisão qualificada, visando formular estratégias empresariais e reforçar a capacidade de competir em um ambiente de constantes mudanças. Nesse sentido, deve-se destacar que, seja no caso de IE ou de IC, a inteligência deriva de uma cultura empresarial proativa. Fuld (2007) ressalta que o trabalho de inteligência é de responsabilidade e oportunidade de todo gestor, devendo estar presente nas suas ações.

\section{Método}

Os resultados apresentados e discutidos neste estudo foram obtidos a partir de uma pesquisa de campo tipo survey realizada no segundo semestre de 2008, dividida em duas etapas. Inicialmente foi realizada uma pesquisa exploratória, de caráter qualitativo, tendo por objetivo levantar subsídios para a construção de um instrumento de coleta de dados composto unicamente por questões fechadas. Após a definição do instrumento, procedeu-se a etapa quantitativa por meio de sua aplicação junto a gestores de uma IES privada da Região Sul do país. O QUADRO 1 apresenta as etapas do estudo acompanhadas de uma breve descrição.

QUADRO 1 Etapas do estudo

\begin{tabular}{c|l|l|l}
\hline Etapa & \multicolumn{1}{|c|}{ Respondentes } & Instrumento & \multicolumn{1}{c}{ Foco } \\
\hline Qualitativa & Diretores de faculdades & $\begin{array}{l}\text { Roteiro semi- } \\
\text { estruturado }\end{array}$ & $\begin{array}{l}\text { Subsídios para construção de } \\
\text { questionário }\end{array}$ \\
\hline Quantitativa & $\begin{array}{l}\text { Diretores de faculdades } \\
\text { Coordenadores de curso }\end{array}$ & $\begin{array}{l}\text { Questionário } \\
\text { estruturado }\end{array}$ & $\begin{array}{l}\text { Atingir os objetivos apresentados na } \\
\text { seção 1. }\end{array}$ \\
\hline
\end{tabular}

Fonte: Elaborado pelos autores (2009).

A etapa qualitativa baseou-se na aplicação de um roteiro semiestruturado visando identificar quais informações representavam o processo de IE na universidade. Foram realizadas oito entrevistas de profundidade com diretores de faculdade da IES investigada, previamente agendadas e sempre conduzidas pelos autores da pesquisa. Nessa etapa, o processo de amostragem caracterizou-se por ser não-probabilístico por julgamento, sendo que o critério fundamental adotado para a seleção dos entrevistados considerou o exercício de cargo de Direção de faculdade. Posteriormente, os resultados dessas entrevistas serviram de subsídio para a elaboração do questionário utilizado na etapa quantitativa.

A primeira versão do questionário da etapa quantitativa era composta por questões de escolha múltipla. Após a realização de um préteste com coordenadores de curso, a versão final do instrumento foi refinada, com ajustes semânticos e de layout, e enviada para 90 gestores da IES. Um total de 46 questionários (51\%) retornou dentro do prazo estabelecido, contemplando todas as áreas do conhecimento da IES (humanas, exatas, biológicas e tecnológicas), sugerindo a representatividade da amostra. 
Nessa etapa quantitativa o procedimento adotado para a coleta de dados foi o de autopreenchimento por parte dos respondentes. Os dados foram organizados em planilha Excel e analisados por meio de técnicas de estatística descritiva, com o apoio do pacote estatístico SPSS (Statistical Package for the Social Sciences) versão 11.5.

\section{Análise e discussão dos resultados}

As análises foram realizadas individualmente por questão e os resultados são apresentados a seguir. A primeira questão solicitava aos respondentes que indicassem quais os três principais elementos de gestão da informação são utilizados para a tomada de decisões em sua faculdade (TAB. 1).

TABELA 1 Principais elementos de gestão da informação para tomada de decisões

\begin{tabular}{lc}
\hline \multicolumn{1}{c}{ Itens } & $\%$ \\
\hline Bancos de dados da instituição & 73,9 \\
Cruzamentos de informações internas & 58,7 \\
Fonte de dados externa & 37,0 \\
Relatórios estatísticos organizados por áreas de apoio & 37,0 \\
Intuição (sensibilidade) & 28,3 \\
Relatórios qualitativos organizados por áreas de apoio & 26,1 \\
Sistemas específicos de Informações Gerenciais & 23,9
\end{tabular}

Nota: Questão de múltipla resposta. Base: 46 gestores.

Fonte: Elaborado pelos autores (2008).

Identificou-se uma grande heterogeneidade nas respostas a essa questão. Apenas dois itens foram assinalados pela maioria dos respondentes, sugerindo a não existência de uma uniformidade na utilização de recursos de gestão da informação. Os resultados da TAB. 1 revelam que fontes internas de informação têm maior preferência dos respondentes em relação às fontes externas. O segundo maior percentual de respostas pode ser justificado pelo fato de que os gestores realizam cruzamentos de dados oriundos de diferentes sistemas institucionais com o objetivo de identificar o perfil do aluno atual ou do aluno potencial.

$\mathrm{Na}$ segunda questão do questionário, os respondentes deveriam apontar cinco tipos de informação consideradas importantes no processo de tomada de decisões gerenciais. A TAB. 2 apresenta a frequência de respostas de cada um dos onze itens que compunham a lista de alternativas. 
TABELA 2 Informações mais importantes para a tomada de decisões gerenciais

\begin{tabular}{lc}
\hline \multicolumn{1}{c}{ Itens } & $\%$ \\
\hline Informações sobre os atuais e potenciais alunos & 78,3 \\
Informações sobre legislações do ensino superior e de órgãos & 60,9 \\
governamentais & 58,7 \\
Informações de cenários futuros & 58,7 \\
Informações sobre a qualidade dos docentes, corpo técnico- & 45,7 \\
administrativo e do curso & 43,5 \\
Informações de indicadores de desempenho & 37,0 \\
Informações de potencial de mercado & 34,8 \\
Informações da concorrência & 32,6 \\
Informações sobre fontes de recursos & 28,3 \\
Informações sobre novas tecnologias & 21,7 \\
Informações de planejamento/marketing & \\
Informações econômicas/financeiras (custos/rentabilidade).
\end{tabular}

Nota: Questão de múltipla resposta. Base: 46 gestores

Fonte: Elaborado pelos autores (2008).

A alternativa com percentual de resposta mais elevado está relacionada à busca de informações sobre alunos atuais e potenciais. Esse resultado mostra-se em sintonia com aqueles da TAB. 1 , onde a busca de informação em bancos de dados da instituição está em primeiro lugar. Para os gestores obterem tais informações é necessário acessar os bancos de dados institucionais.

Informações sobre legislações do ensino superior e de órgãos governamentais, disponibilizadas, sobretudo, nos sites do Ministério da Educação (MEC) e do Instituto Nacional de Estudos e Pesquisas Educacionais Anísio Teixeira (INEP), constituem a segunda fonte de informação mais importante por parte dos gestores. Um resultado que não chega a surpreender porque, em geral, gestores de cursos de ensino superior estão permanentemente sujeitos a cobranças em relação ao Sistema Nacional de Avaliação da Educação Superior (SINAES), mais especificamente, ao Exame Nacional de Desempenho de Estudantes (ENADE). A grande repercussão gerada na mídia, acerca dos conceitos 
derivados do ENADE (RISTOFF; GIOLO, 2006; BITTENCOURT et al., 2008), pode ser explicada pela motivação dos gestores entrevistados para realizar pesquisas periódicas em sites, por exemplo, como o do INEP.

$O$ fato de mais da metade dos gestores buscarem informações sobre cenários futuros revela certa preocupação estratégica com a coleta de informações e meios para assegurar a sustentabilidade e a sobrevivência dos cursos, como foi citado em uma das entrevistas de profundidade realizada com diretores de faculdades. Além de cenários futuros, outra informação considerada importante, com igual percentual de respondentes, é o conhecimento do corpo docente, técnico-administrativo e do curso para a tomada de decisões.

A preocupação com orçamento e indicadores de desempenho aparece a seguir, com menos da metade de respostas. Tal situação pode sugerir a necessidade de se trabalhar, nas faculdades, o desenvolvimento de uma cultura de gestão por indicadores.

Dados sobre potencial de mercado e concorrência apresentam percentuais próximos de $40 \%$ das respostas, repetindo a tendência identificada na TAB. 1, que sugere que os gestores voltam-se mais para o ambiente interno de seu curso do que para o mercado ou para a concorrência. É possível que essa resposta indique que embora os gestores tenham consciência da necessidade de monitorar o mercado, o fato de analisar a concorrência parece ser um elemento menos determinante nessa análise. Esse aspecto poderia merecer uma reflexão maior por parte das instituições de ensino superior, pois, ao que parece, a competição entre as IES não é percebida plenamente pelos gestores, ainda que essa competição seja uma realidade do mercado do ensino superior brasileiro.

Os itens do questionário relacionados a fontes de recursos e novas tecnologias atingiram menos de $35 \%$ das respostas, sendo que os itens com os menores percentuais referem-se às informações econômicas e financeiras $(21,7 \%)$ e informações de planejamento e marketing $(28,3 \%)$. Esses resultados sugerem que, para os entrevistados, as informações mais importantes estão relacionadas ao macroambiente organizacional.

$\mathrm{Na}$ sequência dos resultados da pesquisa, a TAB. 3 refere-se às principais fontes de informações utilizadas pelo gestor para a tomada de decisões gerenciais. Nessa questão o respondente deveria assinalar, em uma lista composta por onze alternativas, as três fontes mais utilizadas na busca por informações que auxiliam suas decisões. 
TABELA 3 Principais fontes de informações utilizadas para tomada de decisão gerencial

\begin{tabular}{lc}
\hline \multicolumn{1}{c}{ Itens } & $\%$ \\
\hline Normas/Legislações & $56,5 \%$ \\
Alunos/Ex-alunos & $54,3 \%$ \\
Especialistas em gestão de ensino superior & $50,0 \%$ \\
Colegas de trabalho & $45,7 \%$ \\
Estudos acadêmicos, de mercado e pesquisas direcionadas & $45,7 \%$ \\
Jornal/Revistas/Periódicos/Livros & $19,6 \%$ \\
Palestras/Convenções/Eventos & $13,0 \%$ \\
Internet/Sites & $10,9 \%$ \\
Orientações superiores & $2,2 \%$ \\
Avaliações internas & $2,2 \%$ \\
Televisão/Rádio & $0,0 \%$ \\
\hline
\end{tabular}

Nota: Questão de múltipla resposta. Base: 46 gestores

Fonte: Elaborado pelos autores (2008).

A TAB. 3 mostra que os cinco primeiros itens formam um grupo mais prevalente, com uma diferença de $11 \%$ do primeiro para o quinto colocado. Normas e legislações, com $56,5 \%$, foi o item considerado como a principal fonte de informação por parte dos gestores. Esse fato não chega a surpreender já que a consulta às normas e à legislação vigente é uma das responsabilidades associadas ao cargo de gestor. Marcon (2008) sustenta que gestores de cursos de graduação utilizam grande parte de seu tempo executando atividades de natureza burocrática.

De forma similar ao que foi revelado nas entrevistas de profundidade, as respostas ao questionário quantitativo demonstraram que, para os entrevistados, as principais fontes de informação são as de caráter pessoal, visto que entre as cinco primeiras fontes de informação, três referem-se à consulta pessoal: alunos/ex-alunos $(54,3 \%)$, especialistas em gestão de ensino superior $(50 \%)$ e colegas de trabalho $(45,7 \%)$.

O percentual de $45,7 \%$ dos gestores entrevistados, o que indica a utilização de estudos acadêmicos, pesquisas de mercado e pesquisas 
direcionadas ressalta a importância da universidade gerar e/ou organizar informação referente ao mercado de ensino superior. Para isso é necessário que haja pessoal técnico responsável por essa tarefa dentro da universidade.

Em relação às alternativas com menor percentual de resposta apresentadas na TAB. 3, os meios de comunicação tradicionais como TV e rádio, não foram citados como principais fontes de informação, embora se pudesse esperar um percentual maior de respostas a essas alternativas.

A quarta e última questão do instrumento de coleta de dados listava seis aspectos referentes ao acesso às informações da instituição. O gestor deveria escolher três consideradas principais em uma lista de seis alternativas. Os resultados são apresentados na TAB. 4.

TABELA 4Aspectos mais importantes para melhorar o acesso às informações

\begin{tabular}{lc}
\hline \multicolumn{1}{c}{ Itens } & $\%$ \\
\hline Cultura institucional na gestão de informações & $87,0 \%$ \\
Definição de indicadores gerenciais & $56,5 \%$ \\
Treinamento específico, quanto à utilização de informações gerenciais & $50,0 \%$ \\
Pessoal especializado em gestão de informações & $45,7 \%$ \\
Tecnologias apropriadas & $43,5 \%$ \\
Sistemas de segurança da informação & $13,0 \%$ \\
\hline
\end{tabular}

Nota: Questão de múltipla resposta. Base: 46 gestores

Fonte: Elaborado pelos autores (2008).

A cultura institucional na gestão de informações foi apontada como o principal aspecto para melhorar o acesso às informações, com $87 \%$, bem acima do segundo item. Essa mesma preocupação também foi salientada nas entrevistas de profundidade com Diretores das faculdades, pois, ainda que tenham uma grande quantidade de informação à disposição, muitos deles não têm o necessário conhecimento sobre como tratá-las ou como utilizá-las em seu benefício. Esse resultado pode justificar, em parte, o fato de metade dos gestores (50\%) considerar importante o terceiro item, voltado ao treinamento específico para a utilização de informações gerenciais.

O segundo aspecto apontado pelos respondentes (definição de indicadores gerenciais) demonstra que os gestores da IES acreditam que a implantação de um sistema de gerenciamento por indicadores poderia melhorar o acesso às informações. Além disso, para $45,7 \%$ dos gestores entrevistados, a contratação de pessoal especializado em gestão de informações poderia ser uma boa opção de investimento por parte da IES.

De uma forma geral, os resultados indicam que a gestão da IES é baseada mais em dados internos do que externos. Informações sobre 
alunos são consideradas mais importantes. Contudo, destaca-se também a importância de informações sobre legislações do ensino superior, cenários e qualidade de docentes e técnicos administrativos. As fontes de informações mais utilizadas são, efetivamente, legislações específicas, os próprios alunos e especialistas em gestão do ensino superior. Os resultados sugerem, também, que para melhorar as práticas de $\mathrm{IE}$, devese trabalhar a cultura da organização orientada para a gestão de informações. Uma política de gestão baseada no uso de indicadores pode ajudar nesse sentido.

\section{Conclusões}

Este estudo apresentou os resultados de uma pesquisa sobre a prática de Inteligência Estratégica (IE) em Instituições de Ensino Superior. A primeira etapa da pesquisa, de caráter qualitativo e executada por meio de entrevistas de profundidade, revelou que os gestores utilizam, de fato, práticas de IE em suas tomadas de decisões, destacando-se 0 planejamento de estratégias para desenvolvimento da suas unidades; a busca de informações sobre o mercado de ensino superior; o monitoramento das ações estratégicas desenvolvidas pelas instituições concorrentes; e a utilização de sistemas de informações gerenciais para organização de dados e a disseminação do exercício da gestão de informação para os seus colaboradores.

$\mathrm{Na}$ segunda etapa da pesquisa, de caráter quantitativo, identificouse que os principais recursos de gestão da informação, apontados pelos entrevistados como os mais utilizados em suas tomadas de decisões, são os dados e informações do ambiente externo da educação superior, além de informações internas provenientes dos bancos de dados da IES. Tratam-se de dados que se apresentam, geralmente, em formato de relatórios estatísticos, organizados por áreas de apoio, o que beneficia os cruzamentos de informações.

Em relação às informações mais importantes para os gestores apoiarem suas decisões gerenciais, destaca-se, em primeiro lugar, as informações sobre os atuais e potenciais alunos e, em seguida, as informações sobre os níveis de qualidade dos docentes, técnicos administrativos e do curso como um todo. Em terceiro lugar estão as informações referentes às legislações do ensino superior e de órgãos governamentais e, por fim, os subsídios para cenários futuros.

Dentre as principais fontes de informações utilizadas pelos gestores entrevistados para tomadas de decisões estratégicas destacam-se as normas e legislações de órgãos regulamentadores do ensino superior, estudos acadêmicos e de mercado e pesquisas específicas. Contudo, os gestores revelam utilizar, principalmente, as fontes pessoais como alunos, ex-alunos, colegas da instituição e especialistas em gestão de ensino superior para suas tomadas de decisões.

Segundo os gestores entrevistados poderiam ser feitas melhorias em relação à aspectos específicos, tais como a ampliação do acesso às 
informações, o desenvolvimento da cultura institucional voltada à gestão de informações na IES, a definição de indicadores gerenciais, além de treinamento específico de pessoal para a utilização de informações gerenciais.

Os resultados da pesquisa aqui apresentada evidenciaram, também, que a IE pode proporcionar para a IES a possibilidade de se antecipar às oportunidades de mercado e de se prevenir quanto a possíveis mudanças no ambiente. A prática de IE também auxilia o aprimoramento da percepção quanto às alterações em cenários futuros, detectando o surgimento de novas tecnologias e tendências, a fim de criar vantagem competitiva em relação a outras instituições. Cabe salientar que futuras pesquisas poderiam ser realizadas com intuito de analisar, de forma mais ampla, processos de IE aplicados em IES. Nesse sentido, variáveis referentes ao impacto do trabalho de IE podem ser analisadas, comparando-se com os resultados específicos de desempenho estratégico e de performance de gestão.

\section{Referências}

ALCARÁ, A. R. et al. Fatores que influenciam o compartilhamento da informação e do conhecimento. Perspectivas em Ciência da Informação, v. 14, n. 1, p. 170-191, 2009.

BATEMAN, T. S.; SNELL. S. A. Administração: construindo vantagem competitiva. São Paulo: Atlas, 1998.

BITTENCOURT, H. R. et al. Uma análise da relação entre os conceitos Enade e IDD. Estudos em Avaliação Educacional, v. 19, p. 247-262, 2008.

BRAGA, M. M.; PEIXOTO, M. C. L.; BOGUTCHI, T. F. Tendências da demanda pelo ensino superior: estudo de caso da UFMG. Cadernos de Pesquisa, n. 113, p. 129-152, 2001.

COSTA, E. A. Gestão Estratégica. São Paulo: Saraiva, 2000

FISCHMANN, A.; ALMEIDA, M. I. Planejamento estratégico na prática. São Paulo: Atlas, 1990.

FREITAS, $\mathrm{H}$. et al. Informação e decisão: sistemas de apoio a seu impacto. Porto Alegre: Ortiz, 1997.

FULD, L. M. Inteligência Competitiva: como se manter à frente dos movimentos da concorrência e do mercado. Rio de Janeiro: Elsevier, 2007.

HITT, M. A.; IRELAND, R. D.; HOSKISSON, R. E. Administração estratégica. São Paulo: Pioneira Thomson Learning, 2002.

INSTITUTO NACIONAL DE ESTUDOS E PESQUISAS EDUCACIONAIS ANÍSIO TEIXEIRA (INEP). Sinopses Estatísticas da Educação Superior. Brasília, DF: [s.n.], 1999-2007. 
JANISSEK-MUNIZ, R.; LESCA, H.; FREITAS, H. Inteligência estratégica antecipativa e coletiva para tomada de decisão. Revista Organização em Contexto, v. 2, n. 4, p. 92-118, 2006.

LAUDON, K. Sistemas de informação. Rio de Janeiro: LTC, 1999.

MARCON, S. R. A. Comportamentos que constituem o trabalho de um gestor de curso de graduação. Tese (Doutorado em Psicologia). Universidade Federal de Santa Catarina, 2008.

MARTINS, A. L. M. A marcha do capitalismo universitário no Brasil nos anos 1990. Avaliação: revista da educação superior, v. 13, n. 3, p. 733743, 2008.

OLIVEIRA, D. P. R. Estratégia empresarial e vantagem competitiva. São Paulo: Atlas, 2001.

PRADO, L. J. Inteligência competitiva como administrar um mar de informações. 2007. (Série Gestão Empresarial). Disponível em: < http://lauroprado.tripod.com/ezine/>. Acesso em: 11 nov. 2007.

REZENDE, D. A.; ABREU, A. F. Tecnologia da informação aplicada a sistemas de informação empresariais. 3 ed. São Paulo: Atlas, 2003.

RISTOFF, D.; GIOLO, J. O Sinaes como Sistema. RBPG, v. 3, n. 6, p. 193213, 2006.

SOCIETY OF COMPETITIVE INTELLIGENCE PROFESSIONALS (SCIP). Intelligence Competitive. 2005. Disponível em: <http://www.scip.org>. Acesso em: 11 nov. 2007.

VAITSMAN, H. S. Inteligência empresarial: atacando e defendendo. Rio de Janeiro: Interciência, 2001.

WRIGHT, P.; KROLL, M. J.; PARNELL, J. Administração estratégica. São Paulo: Atlas, 2000. 